\title{
Role of Ki-67 and Argyrophilic Nucleolar Organiser Region (AgNoR) Counts in Squamous Cell Carcinoma
}

\author{
Neelima Verma ${ }^{1}$, Suman Lata Verma ${ }^{2}$, Shadab Ahmad ${ }^{3 *}$, Neena Gupta ${ }^{4}$ \\ ${ }^{1}$ Professor, Department of Pathology, GSVM Medical College Kanpur, India \\ ${ }^{2}$ Professor and Head, Department of Pathology, GSVM Medical College, Kanpur, India \\ ${ }^{3}$ Junior Resident, Department of Pathology, GSVM Medical College Kanpur, India \\ ${ }^{4}$ Professor, Department of Obstetrics and Gynecology, GSVM Medical College Kanpur, India
}

\begin{abstract}
*Address for Correspondence: Dr. Shadab Ahmad, Junior Resident, Department of Pathology, G. S. V. M. Medical College, Kanpur, India
\end{abstract}

E-mail: shadab1005ansari@gmail.com

Received: 11 Sep 2021/ Revised: 14 Nov 2021/ Accepted: 27 Dec 2021

\begin{abstract}
Background: Squamous cell carcinoma of the cervix is the most common subtype of cervical cancer and it usually accounts for 80$90 \%$ of the cases. These carcinomas mostly grow at the squamocolumnar junction (SCJ). The tumour tends to grow outward showing an exophytic growth especially at the squamocolumnar junction outside the external uterine wall whereas cancer tends to grow along the cervical canal showing an endophytic growth pattern where the SCJ is located within the cervical canal. This study has experimentally shown the role of AgNOR and Ki-67 scores in tumour proliferation.

Methods: Cervical specimens were obtained and prepared in a specified fashion as described. The samples were dewaxed and staining was done with AgNOR. Then, the AgNOR was counted and scoring was done. Again, the samples were also stained with Ki-67.

Results: This study has included 235 cases from the Department of Pathology of Ganesh Shankar Vidyarthi Memorial Medical College, Kanpur, India. The mean value of AgNOR count in the Control group came to be $10.62 \pm 0.45$ while the mean AgNOR count was found to be $10.62 \pm 0.45,15.10 \pm 0.79,18.39 \pm 0.67,19.75 \pm 0.74$ and $19.59 \pm 0.59$ for in-Situ SCC, well-differentiated SCC, moderately differentiated SCC, poorly differentiated SCC, SCC with basaloid differentiation, respectively.

Conclusion: The study confirmed that AgNOR count increases with a higher grade of malignancy. It was concluded that AgNOR and Ki-67 scores can be used as an efficient predictor of tumour proliferation.
\end{abstract}

Key-words: AGNOR, Cervical carcinoma, in-Situ SCC, Ki-67, Squamouscell carcinoma

\section{INTRODUCTION}

Squamous cell carcinoma of the cervix is the most common subtype of cervical cancer ${ }^{[1]}$ and it usually accounts for $80-90 \%$ of the cases ${ }^{[2]}$. These carcinomas mostly grow at the squamocolumnar junction (SCJ) ${ }^{[1]}$. The tumour tends to grow outward showing an exophytic growth pattern in younger women where the squamocolumnar junction is located outside the external uterine wall, whereas cancer tends to grow along the

\section{How to cite this article}

Verma N, Verma SL, Ahmad S, Gupta N. Role of Ki-67 and Argyrophilic Nucleolar Organiser Region (AgNoR) Counts in Squamous Cell Carcinoma. SSR Inst. Int. J. Life Sci., 2022; 8(1): 2953-2961.

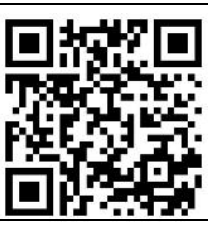

Access this article online https://iijls.com/ cervical canal showing an endophytic growth pattern, where the SCJ is located within the cervical canal. Most tumours arise from a pre-cancerous disease called highgrade squamous intraepithelial lesion (HSIL). Most of these carcinomas are a result of the normal squamous cells in the cervix becoming infected with a high-risk type of virus called the human papillomavirus (HPV) ${ }^{[3,4]}$. Various investigations of the study of disease transmission of cervical malignant tumours have shown a solid relationship with, conjugal and sexual patterns. Even though it is grounded that women with numerous accomplices and early ages at first intercourses are in high danger, though less information is known regarding how these factors have their effects. Recent examinations reveal that steady partners and frequent intercourse may further enhance the risk, supporting hypotheses regarding a vulnerable period of the cervix 
and a requirement for rehashed openness to an infectious agent. It is currently broadly acknowledged that HPV is a major infectious etiological agent. Other risk factors for cervical malignant growth include cigarette smoking, oral contraceptive use, and certain malnutrition. Recent investigations have shown that there is a need to highlight not only the female factor but also the male factor. To conclude this, genetic factors, bacterial infections, viral infections, smoking, and sexual habits are some of the known risk factors ${ }^{[4,5]}$. Ki-67 is broadly used in the routine pathological investigation as a proliferation marker as its expression is strongly associated with tumor cell proliferation and growth ${ }^{[6]}$. The assessment of the biopsies from the cancer patients is known to be established with nuclear protein Ki-67 ${ }^{[7]}$. It has been clinically displayed to relate with metastasis and clinical phases of cancer. The Ki-67 marking index is an autonomous prognostic component for survival rate, which incorporates all stages and grade classifications. The arrest of cell proliferation has been achieved by blocking Ki-67 either by microinjection of antibodies or through the use of antisense oligonucleotides ${ }^{[8]}$. The number of nuclei staining positively for these markers of proliferation can be estimated by simple counting or can be measured in an image analysis system. In cervical intraepithelial neoplasia, both proliferating cell nuclear agent and Ki-67 expressions are increased in the upper levels of the cervical epithelium as compared to normal cervices ${ }^{[9]}$. And therefore it is thought that this staining technique especially Ki-67 might be very helpful in distinguishing cervical intraepithelial neoplasia from non-neoplastic lesions. Expanding proof shows that Ki76 might be a viable objective in disease treatment ${ }^{[10]}$.

Nucleolar organizer regions are described as nucleolar components which contain a set of argyrophilic proteins, preferentially stained by silver methods. After the process of staining, these NORs can be easily recognized as black dots throughout the nucleolar area. This occurs due to a group of nucleolar proteins which have a high affinity for silver (AgNOR proteins) ${ }^{[11]}$. The AgNOR method has been applied in tumor pathology for both diagnostic and prognostic purposes for the past 12 years. These proteins accumulate highly in proliferating cells whereas their expression is very low in non-proliferating cells ${ }^{[12]}$. Ki-67 immunostaining was known to be performed on 48 cases of squamous cell carcinoma of the cervix and AgNOR silver staining on 29 cases. Though no correlation was found between the method of staining and tumor stages, they correlated on a histological level. The presence of a high Ki-67 score but not a high AgNOR count was linked to early recurrence [13].

\section{MATERIALS AND METHODS}

Study type and Source of data- The type of study is Retrospective Cohort. The samples and data were obtained from G.S.V.M. Medical College, Kanpur, Uttar Pradesh, India. This study included retrospective cases from March 2019 to August 2021.

Sample collection and preparation- The cervical biopsies or hysterectomy specimens were collected from the Department of Pathology, G.S.V.M. medical college, Kanpur, Uttar Pradesh. Fixation of cervical biopsies or hysterectomy specimens was performed with buffered formalin. A complete gross examination of the cervical biopsy and hysterectomy specimen was completed, including a look at the cut surface as well as the outer surface of the tissue. In histology biopsy tissue, tissue pieces were processed as a whole; in hysterectomy specimens, tissues were sectioned and processed as two lips of the cervix.

Inclusion criteria- The patients, who were enrolled in G.S.V.M. medical college, Kanpur, Uttar Pradesh and were suspected of cervical carcinoma. The control group was selected from random patients, whose cervical biopsy was done.

Exclusion criteria- The patients who discontinued their management in G.S.V.M. medical college. The patients whose, AgNOR staining score came to be either 0 or 1.

Procedure for processing tissue- The tissue was placed in an automatic tissue processor. On Day 1, different percentages of alcohol are taken and processed for around 2 hours to obtain the outcome. On Day 2, different materials like alcohol, xylene, and wax are used in this procedure and are processed for around two hours to obtain the final result. On Day 3, a thermostatically controlled water bath was used in which the cassettes were left for 15 minutes. From paraffin blocks, microtomes were used to cut sections of four microns thickness. 
These cut sections were then placed on a hot plate at a temperature of 65 degrees centigrade. After this, the sections were stained with routinely used $\mathrm{H}$ and $\mathrm{E}$ stain. Hematoxylin and Eosin stains are used for staining the sections.

To make a hematoxylin solution, you need alcohol, distilled water, mercuric oxide, hematoxylin, potassium alum or sodium iodate, and glacial acetic acid in certain amounts. The sections are stained for 30 seconds in 1\% eosin. After staining the sections are washed for 1-5 minutes under tap water. They are then dehydrated by alcohol, xylene is used for clearing the section, and then the sections are mounted on DPX. Staining the section resulted in Black or blue colored nuclei, shades of pink in the cytoplasm, red or orange colored RBC.

Staining of AgNOR- The paraffin wax of 4 microns thickness section that is fixed with formalin is used for the staining procedure. Xylene was used for the deparaffinization of the sections. After that, the section was treated with absolute alcohol. The sections were then put in a mixture of 1:3 ratio of acetic acid and alcohol for nearly 5 minutes. The solution was then put in absolute alcohol. Then they are dipped in $80 \%$ and $70 \%$ of graded alcohol respectively. After they are dipped in alcohol they are washed in distilled water for about 5 minutes. The sections are then kept for incubation in a freshly prepared working solution. They are then washed for nearly 5 minutes under distilled water. Finally, the sections are dehydrated in absolute alcohol. The sections are then cleared with xylene. After that, they are mounted in DPX. Lastly, AgNOR was stained with Dark brown or black dots while the background was yellow stained.

Counting of AgNOR- A representative area is selected by examining the stained sections under a low-power microscope. An oil immersion lens of 100x and an eyepiece of 10x is used for counting AgNORs with a total magnification of $1000 x .30$ cells were counted in every case and at last, a mean value is calculated. Under a lowpower microscope, the AgNORs are present as clusters that contain discernible dots and they also contain individual satellites. The clusters that contain discernible dots represent the nucleoli.

Parameters used in AgNOR scoring- The scores and their respective parameters are given below (Table 1 ).
Table 2: Staining patterns of cases with its score

\begin{tabular}{cc}
\hline Parameters & score \\
\hline 1. Estimated number of AgNOR & 1 \\
per cellLess than 2 & 2 \\
$2-5$ & 3 \\
More than 5 & 1 \\
2. Variation in the size and shape & \\
of satellite cell (each & \\
scored) Uniform & Moderate variation \\
Marked variation & 2 \\
3. Variation in size and shape of & 3 \\
the cluster (each & 1 \\
scored) Uniform & \\
Moderate variation & 2 \\
Marked variation & 3 \\
\hline
\end{tabular}

The mean value of each case is calculated after counting the total number of AgNOR dots. Then the standard deviations were calculated after obtaining the mean value of all the cases.

Staining procedure of $\mathrm{Ki}-67-$ This procedure is completed in 3 steps. The slide is pre-coated using a poly-L-Lysine a suitable adhesive, the preparation of the immunostain and the preparation of chromogen.

Preparation of reagents and buffer- Tris-buffer saline of $7.6 \mathrm{pH}$ is prepared in $750 \mathrm{ml}$ of distilled water, 8.5 grams of sodium chloride, and 6.06 grams of tris (hydroxyl) methylamine as dissolved using $50 \%$ of $\mathrm{HCl}$, the $\mathrm{pH}$ of the solution is adjusted to 7.6. The final volume of the solution is then adjusted to one liter. Citrate buffer $10 \mathrm{mM}$ of $\mathrm{pH}$ 6. Stock solution A contains $0.1 \mathrm{~m}$ of citric acid. Stock solution $B$ contains $0.1 \mathrm{~m}$ od $=\mathrm{f}$ sodium citrate that is stored at a temperature of 2-8 degrees centigrade. The working solution contains $9 \mathrm{ml}$ of solution $A+4 \mathrm{ml}$ of solution $B$ and the final volume of the solution was adjusted to $500 \mathrm{ml}$.

\section{Steps of immuno-staining}

Deparaffinization-The sectioned paraffin wax was put on a hot plate for 2 minutes so that the wax melts. The sections were washed under tap water and then are processed to the next step. The sections embedded in formalin require treatment to remove the over-fixed antigenic sites. The effective method for the retrieval of the antigen is microwave treatment. Retrieval of the epitope by usingcitrate buffer is for the retrieval of the 
epitope. The citrate buffer is placed in a glass Coplin or plastic jar. The slides were immersed vertically in the jar. Then the jar is placed in the microwave for 5 minutes for 3 cycles of boiling. The buffer is then brought to a boiling temperature and it was confirmed that the slides were not dried. The slides are brought to a cooling temperature for 20 minutes. The sections were rinsed in a washing buffer of 2 changes for 2 minutes each. To block the binding of immunoglobulin in a non-specific way, the sections were incubated for 30 minutes in a normal horse serum blocking solution. After the completion of incubation, the slides were washed for 2 minutes in Tris-buffer saline. The sections were incubated for 10 minutes in peroxide blocking solutions to prevent the activity of endogenous peroxidase. The slides were then rinsed in 2 changes of buffer for 2 minutes. The slides were incubated at room temperature for one hour with the mouse monoclonal or rabbit monoclonal antihuman B cell, Ki-67. The antibody is removed and the slides were placed for 5 minutes in buffer saline for washing. The slides were then incubated in a moist chamber for 80 minutes with a superenhancer. The slides were rinsed for 2 changes in a buffer for 2 minutes each. The slides were incubated and diluted at room temperature for 30 minutes in horseradish peroxidase-streptavidin. The complex was removed off and the slide is placed for 5 minutes in buffer saline. Substrate or chromogen: the slides were incubated for 5-10 minutes in diaminobenzidine peroxide. The slides were then washed in distilled water. Gills hematoxylin solution was used for counterstaining the slide. The dehydration of the slide is done through 95 percent of alcohol for 2 minutes, 100 percent of ethanol of 2 changes for 3 minutes each.The slides are cleared using the xylene and the coverslip is placed over it with permanent mounting.

Interpretation (Ki-67 score)- The Ki-67 labelling index was calculated in percentage as the immune stained cells were divided by the total number of cells present in the evaluated area. A 1000x magnification of oil immersion is used for counting the cells. Averages of 10 fields were taken and their standard and mean deviations were calculated. An attempt is made in looking for the stained positive cells in the maximum density area.

Patterns of staining- Cases with 0-1 scoring were not included in the study.
Table 2: Staining patterns of cases with its score

\begin{tabular}{cc}
\hline Score & Staining Pattern \\
\hline 0 & Staining is not seen \\
1 & Less than 10 percent of positive cells are seen \\
2 & $10-50$ percent of positive cells are seen \\
3 & Greater than 50 percent of positive cells are \\
\end{tabular}

\section{RESULTS}

This study considered 235 cases from the Department of Pathology of Ganesh Shankar Vidyarthi Memorial Medical College, Kanpur, India. The following observations were made and analyzed. Figure 1 below shows the age distribution of the patients included in this study. The table shows the maximum number of cases ( $n=132 ; 56.17 \%$ ) among the age group of 41 years to 60 years. The lowest number of patients was found in the age group of $81-100$ years old.

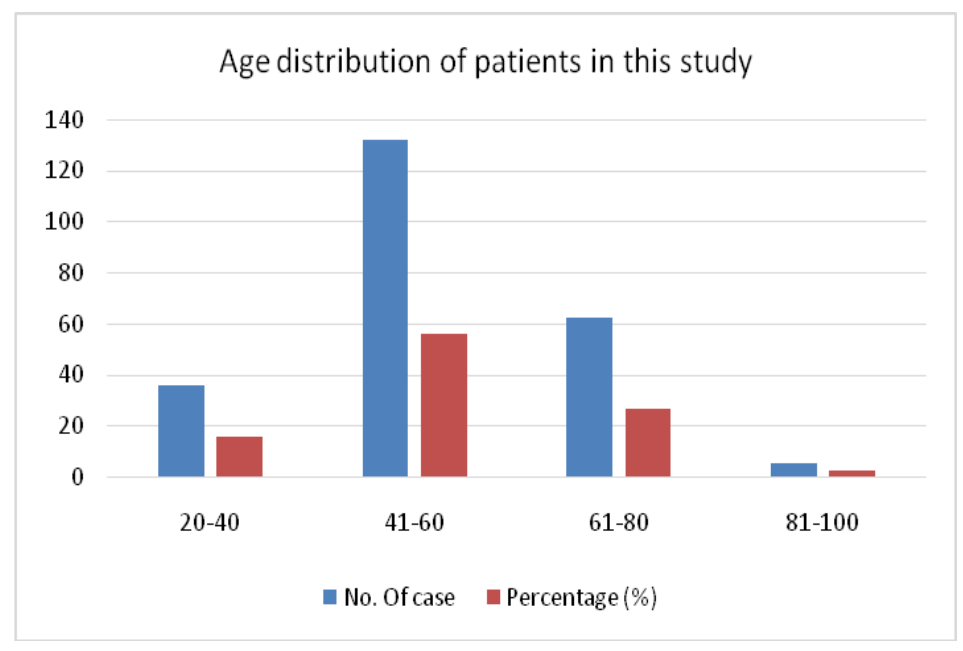

Fig. 1: Age-wise distribution of cases in this study

Table 3 below shows the number of patients in each type of carcinoma for each age group. The table below also shows the percentage of the total patients in each category. The study found that $7.65 \%$ of the patients are having In Situ SCC, while well-differentiated type, moderately-differentiated type and poorly-differentiated type accounted for $29.36 \%, 20.85 \%$ and $18.29 \%$, respectively (Table 3 ). 
Table 3: Age-wise distribution with the type of carcinoma

\begin{tabular}{cccccc}
\hline Type & $\mathbf{2 0 - 4 0}(\mathbf{y r})$ & $\mathbf{4 1 - 6 0}(\mathbf{y r})$ & $\mathbf{6 0 - 8 0}(\mathbf{y r})$ & $\mathbf{8 1 - 1 0 0}(\mathbf{y r})$ & Total \\
\hline In situ SCC & 1 & 15 & 2 & 0 & 18 \\
Total patients (\%) & 0.4 & 6.38 & 0.85 & 0 & 7.65 \\
Well diff. SCC & 16 & 48 & 5 & 0 & 69 \\
Total patients (\%) & 6.8 & 20.42 & 2.12 & 0 & 29.36 \\
Mod. Dif. SCC & 8 & 16 & 22 & 3 & 49 \\
Total patients (\%) & 3.4 & 6.8 & 9.36 & 1.27 & 20.85 \\
Poorly diff. SCC & 0 & 26 & 15 & 2 & 43 \\
Total patients (\%) & 0 & 11.06 & 6.38 & 0.85 & 18.29 \\
SCC with basaloid diff. & 11 & 27 & 26 & 0 & 64 \\
Total patients (\%) & 4.68 & 11.48 & 11.06 & 0 & 27.23 \\
Total & 36 & 132 & 62 & 5 & 235 \\
Total patients (\%) & 15.31 & 56.17 & 26.38 & 2.12 & 100 \\
\hline
\end{tabular}

Fig. 2 shows the number of patients in this study, whose lesion is precancerous and those, who have a malignant lesion. 162 patients are having large cell invasive SCC and 55 patients have small cell invasive SCC. There are 18 patients with the precancerous lesion. Table 3 represents the summary of the distribution of tumour grades in the patient population.

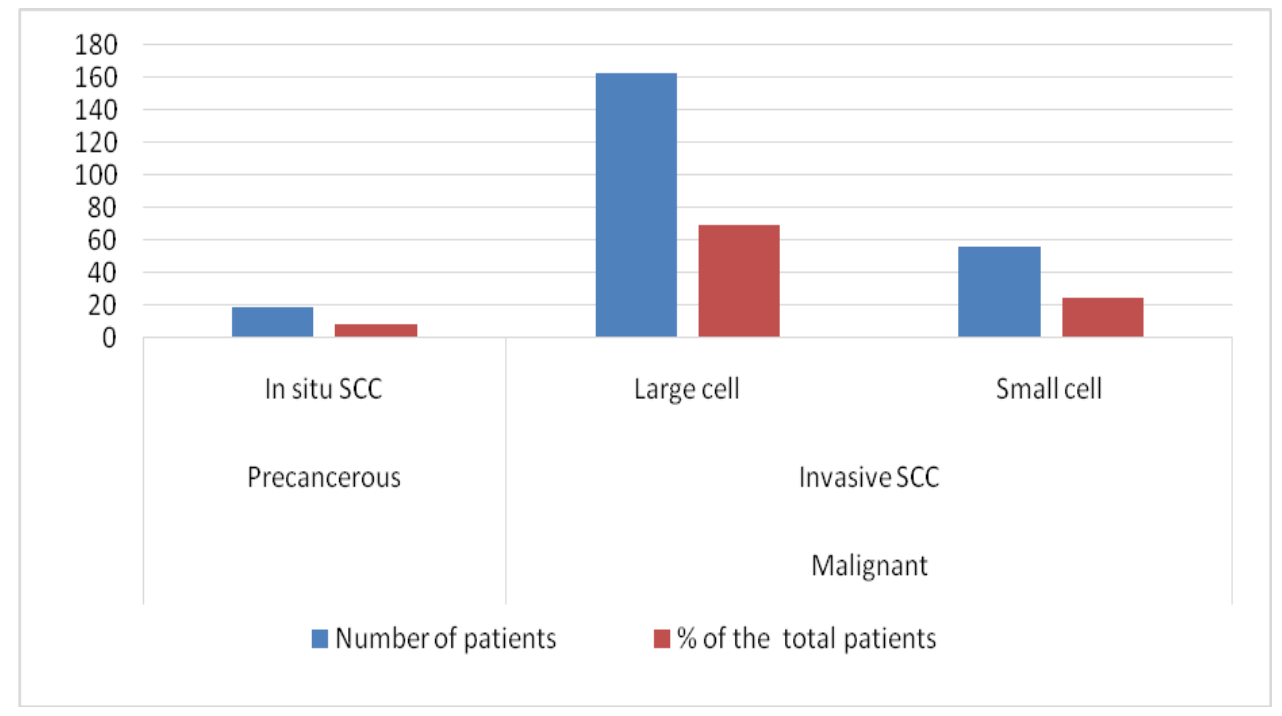

Fig. 2: Distribution of tumor grade in the patients

The study also found that $45.10 \%$ of the total patients are post-menopausal. It is again shown that $14.89 \%$ of the total patients are post-menopausal with poorly diff. SCC, the lesion, which is the most prevalent among postmenopausal patients. This is followed by Mod. Dif. SCC (13.19\%), Well diff. SCC (10.63\%) and SCC with basaloid diff. (6.38\%). Table 4 presents the findings of tumor gradings in postmenopausal patients.
Table 4: The number of post-menopausal patients in each category of tumor grading

\begin{tabular}{ccc}
\hline Type & Total & $\begin{array}{c}\text { Number of } \\
\text { postmenopausal } \\
\text { patients }\end{array}$ \\
\hline In situ SCC & 18 & 0 \\
Total patients (\%) & 7.65 & 0
\end{tabular}




\begin{tabular}{ccc} 
Well diff. SCC & 69 & 25 \\
Total patients (\%) & 29.36 & 10.63 \\
Mod. Dif. SCC & 49 & 31 \\
Total patients (\%) & 20.85 & 13.19 \\
Poorly diff. SCC & 43 & 35 \\
Total patients (\%) & 18.29 & 14.89 \\
SCC with basaloid diff. & 64 & 15 \\
Total patients (\%) & 27.23 & 6.38 \\
Total & 235 & 106 \\
Total patients (\%) & 100 & 45.10 \\
\hline
\end{tabular}

Table 5 shows the average (mean) AgNOR counts in each grade of malignancy along with respective standard deviation (SD). The mean value of the Control group came to be $10.62 \pm 0.45$ while the mean AgNOR count was found to be $10.62 \pm 0.45,15.10 \pm 0.79,18.39 \pm 0.67$, $19.75 \pm 0.74$ and $19.59 \pm 0.59$ for in-Situ SCC, welldifferentiated SCC, moderately differentiated SCC, poorly differentiated SCC, SCC with basaloid differentiation, respectively. Table 5 also shows Ki-67 labelling index in each grading of tumor. Ki-67 score in the control group was found to be $12.71 \pm 0.45$. With the increase of grading of the carcinoma, the mean value of Ki-67 score increases as it can be seen from in Situ SCC with the score of $25.11 \pm 0.65$, the lowest one. This is followed by well-differentiated SCC $(43.00 \pm 1.25)$ while for moderately differentiated SCC and poorly differentiated SCC, the score came to be $48.62 \pm 1.56$ and $56.54 \pm 0.97$ respectively. Unlike AgNOR counts in basaloid differentiation grade, Ki-67 score was found lesser (51.72 \pm 0.71$)$ as compared to poorly differentiated SCC (56.54 0.97$)$.

Table 5: Mean AgNOR counts and Ki-67 score in each grade of cervical carcinoma

\begin{tabular}{lcrc}
\hline Grade & $\begin{array}{c}\text { No. of } \\
\text { cases }\end{array}$ & $\begin{array}{c}\text { Mean } \\
\text { AgNOR \& } \\
\text { respective SD }\end{array}$ & $\begin{array}{c}\text { Ki-67 } \\
\text { score } \pm S D\end{array}$ \\
\hline Control & 60 & $10.62 \pm 0.45$ & $12.71 \pm 0.45$ \\
In Situ SCC & 18 & $13.97 \pm 0.65$ & $25.11 \pm 0.65$
\end{tabular}
Well-
69
$15.10 \pm 0.79$
$43.00 \pm 1.25$

differentiated

Moderately

49

$18.39 \pm 0.67$

$48.62 \pm 1.56$

differentiated

\begin{tabular}{|c|c|c|}
\hline Poorly & 43 & $19.75 \pm 0.74$ \\
\hline
\end{tabular}

differentiated

SCC with

64

$19.59 \pm 0.59 \quad 51.72 \pm 0.71$

basaloid

differentiation

\section{DISCUSSION}

Potential doubling time has been proposed as a pretreatment estimator of intra-treatment tumor proliferative capacity. In this study, Ki-67 immunostaining and AgNOR silver staining were examined as a possible alternative method by comparing them with the doubling time using different cell lines under varying growth conditions, in vitro. A strong correlation was found between these scores. Both these had a significant inverse relation with cell doubling time, potential doubling time, length of S-phase. These values along with other measures suggest their use as a potential indicator of proliferative activity ${ }^{[14]}$. In our present study, the primary conclusion was that the relationship of AgNOR and Ki-67 immunostaining can be considered for determining the proliferative activity of tumor.

All pre-treatment biopsy values were analyzed for tumor proliferative compartment by evaluation of Ki-67 antigen expression and argyrophilic nucleolar organizer region (AgNOR) counts. Growth factor analysis was done by analyzing for expression of epidermal growth factor (EGF), epidermal growth factor receptor (EGF-R), and transforming growth factors alpha and beta (TGF $\alpha$, TGF $\beta$ ). A total of 152 patients were evaluated and a correlation was obtained between the pre-treatment status of the tumour-growth-fraction-associated markers and clinical outcome following radiotherapy. Such patients were either disease-free (group 1, $n=106$ ) recurrent disease (group 2, n=46) at a 16-month followup. Pre-treatment analysis of AgNOR ( $r=-0.517$, $p=0.0000)$. This may be due to the effect of cell proliferation. Lower AgNOR counts were significantly associated with recurrent tumors, suggesting that increased proliferative activity may be a positive prognostic indicator. Similar results were also obtained for the other proliferation-associated marker Ki-67 ( $r=-$ $0.443, P=0.0000)$. Expression of $E G F$ and $E G F-R$ also 
showed significant pre-treatment correlations with the final disease outcome $(r=0.248, P=0.031$ and $r=0.503$, $\mathrm{P}=0.0000$, respectively). Both these markers were expressed more by patients belonging to group2. Group 1 patients showed mostly mild to moderate expression whilst most group 2 patients were negative for the growth factor. It, therefore, appears that tumors with high AgNOR counts and Ki-67 index, along with expression of the two types of transforming growth factor, responded well to radiotherapy ${ }^{[15]}$.

Another study was aimed at assessing the cell proliferation in various grades of squamous cell carcinoma of the cervix using two proliferation markers that is AgNOR and Ki-67. 18 biopsy sections of various grades of squamous cell carcinoma were taken. AgNORs staining was done by single-step staining technique using gelatin. Ki-67 immunostaining was done by the AvidinBiotin technique using DAB as a chromogen. The highest mean number of AgNORs was found in poorly differentiated squamous cell carcinoma and statistically, a significant difference was observed between well and poorly differentiated squamous cell carcinoma and between moderately and poorly differentiated squamous cell carcinoma. The highest mean Ki-67 was seen in poorly differentiated squamous cell carcinoma. When observation was made between histological grade and Ki-67 and AgNORs separately but no statistical correlation was found between $\mathrm{Ki}-67$ and AgNOR counts. AgNORs and Ki-67 are simple and easily performed techniques which help determine the management strategy of the patient ${ }^{[16]}$.

Uterine smooth muscle tumors are the most common human neoplasms, which are divided into malignant and benign. There is another set of lesions called smooth muscle tumor of uncertain malignant potential (STUMP) which does not fit into either of these categories. 21 cases of the above-mentioned group tumors were randomly collected and were stained with Ki-67 and Argyrophilic nucleolar organizing regions. Ki-67 was expressed in $63.15 \%$ of leiomyosarcomas, $4.76 \%$ of STUMPs, and $0 \%$ of the leiomyoma group.Ki-67 expression was 2.55 $\pm 0.03,2.55$. 0.66, 4.04, and $8.12 \pm 0.13$ in leiomyoma, STUMP, and leiomyosarcoma, respectively. Differences among the groups were observed ${ }^{[17]}$. Fifty cases each of cytologically diagnosed normal, inflammatory, different grades of squamous intraepithelial lesions (SIL), LSIL, HSIL, and squamous cell carcinoma were registered. In normal smears, the number of total AgNOR dots ranged from 1 to 2, and in $41.8 \%$ of cases, the dots were pleomorphic. In the inflammatory smears, the number of AgNOR dots varied from 2 to 3, and the percentage of pleomorphic dots was $45.3 \%$ in the case of HSIL, AgNOR dots varied from 5 to 7 with $56.7 \%$ pleomorphic dots. In frank cancer cases, the AgNOR dots were 8-10 with 62.9\% pleomorphic dots. The correlation of cell counts of both pleomorphic and single dots with disease severity was positive and significant. This reveals that AgNOR pleomorphism increases with the severity of cytopathological lesions of the cervix. Hence, pleomorphic dots can be very useful in determining and establishing the prognosis of the disease and treatment planning ${ }^{[18-20]}$.

Diagnosis of atypical cells of unspecified importance (ASCUS and AGUS) might help clinicians carry out further diagnostic procedures to determine the process going on in the female reproductive system. Qualifying the atypical cells as neoplastic type or reactive type was done with proliferative potential cells in cytological smears of Ki-67 and AgNOR. ASCUS had the highest average number of AgNORs in the cell nucleus. The ratio of AgNORs to that of the cell nucleus was found to be highest in HSIL and cancer cells, and the east was found in ASCUS. Thus analysis of AgNORs makes it easier to differentiate ASCUS and LSIL cells from HSIL and neoplastic ones ${ }^{[21-24]}$. There is also a well-established association of cervical cancer with education, area of residence, using old/unhygienic sanitary napkins, young age at marriage, washing genitalia after sexual intercourse, number of husband's partners, and availability of health care services. Not bathing every day and also during menstruation was found to be a risk ${ }^{[10]}$.

\section{CONCLUSIONS}

The study confirmed that AgNOR count increases with higher grade of malignancy. Distilled water also proved to bring better results when used for washing the sample. Again, the study inferred that Ki-67 score increases with increased malignancy grade.

The inter-relationship between AgNOR counts and Ki-67 score is a suitable and efficient indicator of proliferative activity. Hence, AgNOR and Ki-67 score can be used as an efficient predictor of tumor proliferation. 


\section{CONTRIBUTION OF AUTHORS}

Research concept- Dr. Neelima Verma

Research design- Dr. Neelima Verma, Dr. Suman Lata Verma

Supervision- Dr. Neelima Verma, Dr. Suman Lata Verma, Dr. Neena Gupta

Materials- Dr. Shadab Ahmad

Data collection- Dr. Shadab Ahmad, Dr. Neena Gupta

Data analysis and Interpretation- Dr. Shadab Ahmad

Literature search- Dr. Shadab Ahmad

Writing article- Dr. Shadab Ahmad

Critical review- Dr. NeelimaVerma

Article editing- Dr. NeelimaVerma, Dr. Shadab Ahmad

Final approval- Dr. NeelimaVerma, Dr. Shadab Ahmad, Dr.

Suman Lata Verma

\section{REFERENCES}

[1] Singh N, Horn LC. Squamous Cell Carcinoma of the Cervix. In: Herrington C. (eds) Pathology of the Cervix. Essentials of Diagnostic Gynecological Pathology, vol 3. Springer, 2017. doi: 10.1007/9783-319-51257-0_7.

[2] Mocarska A, Starosławska E, Zelazowska-Ciesliska I, Łosicki M, Stasiewicz D, et al. Epidemiology and risk factors of the cervical squamous cell carcinoma. Paul Mercury Med., 2012; 33(194): 101-06.

[3] Sundström K, Eloranta S, Sparén $P$, Arnheim DL, Gunnell $A$, et al. Prospective study of human papillomavirus (HPV) types, HPV persistence, and risk of squamous cell carcinoma of the cervix. Cancer Epidemiol Biomarkers Prev., 2010; 19(10): 2469-78. doi: 10.1158/1055-9965.

[4] Sowjanya AP, Jain M, Poli UR, Padma S, Das M, et al. Prevalence and distribution of high-risk human papilloma virus (HPV) types in invasive squamous cell carcinoma of the cervix and normal women in Andhra Pradesh, India. BMC Infect Dis., 2005; 22(5): 116. doi: 10.1186/1471-2334-5-116.

[5] El-Mofty SK, Zhang MQ, Davila RM. Histologic identification of human papillomavirus (HPV)-related squamous cell carcinoma in cervical lymph nodes: a reliable predictor of the site of an occult head and neck primary carcinoma. Head Neck Pathol., 2008; 2(3): 163-68. doi: 10.1007/s12105-008-0066-1.

[6] Chatterjee SK, Zetter BR. Cancer biomarkers: knowing the present and predicting the future. Future Oncol., 2005; 1: 37-50.
[7] Scott RJ, Hall PA, Haldane JS. A comparison of immunohistochemical markers of cell proliferation with experimentally determined growth fraction. J. Pathol., 1991; 165: 173-78.

[8] Teixeira M, de Magalhães FT, Pardal-de-Oliveira F. Squamous-cell carcinoma of the endometrium and cervix. Int J Gynaecol Obstet., 1991; 35(2): 169-73. doi: 10.1016/0020-7292(91)90822-m.

[9] Felix JC, Lonky NM, Tamura K. Aberrant expression of E-cadherin in cervical intraepithelial neoplasia correlates with a false negativePapanicolaou smear. Am J Obstet Gynecol., 2002; 186: 1308-14.

[10]Oka K, Hoshi T, Arai T. Prognostic significance of the PC10 index as a prospective assay for cervical cancer treated with radiation therapy alone. Cancer, 1992; 70: 1545-50.

[11]Crocker J, Boldy AR, Egan MJ. How should we count AgNORs? Proposal for a standardized approach. J Pathol., 1989; $\quad 158: \quad 185-88$. doi: 10.1002/path.1711580303.

[12]Srivastava A, Srivastava S, Bansal C, Misra, J. Diagnostic importance of AgNORpleomorphism in cervical carcinogenesis. CancerMedSci., 2013; 7: 287. doi: 10.3332/ecancer.2013.287.

[13]Chung TKH, Cheung TH, Wong FWS, Wong YF. Ki67 and AgNORs Staining in Squamous Cell Carcinoma of the Cervix: A Comparison. GynecolObstet Invest., 1994; 37(2): 127-29. doi: 10.1159/000292540.

[14]Dong H, Bertler C, Schneider E, Ritter MA. Assessment of cell proliferation by AgNOR scores and Ki-67 labeling indices and comparison with potential doubling times. Cytom., 1997; 28(4): 28088.

[15]Pillai MR, Jayaprakash PG, Nair MK. Tumourproliferative fraction and growth factor expression as markers of tumour response to radiotherapy in cancer of the uterine cervix. J Cancer Res ClinOncol., 1998; 124(8): 456-61. doi: 10.1007/s004320050199.

[16]Pahuja S, Choudhury M, Gupta, U. Proliferation in squamous cell carcinoma of cervix: a comparative assessment by two markers. Ind J Path Microbiol., 2003; 46(4): 585-88.

[17] Mayerhofer K, Lozanov P, Bodner K, Bodner-Adler B, Kimberger O, et al. Ki-67 expression in patients with uterine leiomyomas, uterine smooth muscle tumors of uncertain malignant potential (STUMP) and uterine leiomyosarcomas (LMS). Acta Obstet Gynecol 
Scand., 2004; 83(11): 1085-88. doi: 10.1111/j.00016349.2004.00502.x. PMID: 15488127.

[18]Dong H, Bertler C, Schneider E, Ritter MA. Assessment of cell proliferation by AgNOR scores and Ki-67 labeling indices and comparison with potential doubling times, Cytom., 1997; 28: 280-88.

[19]Pich A, Valente G, Azzoni L, Stramignoni A, Margaria $E$, et al. Argyrophilic Nuclear Organizer Region Counts and Ki-67 Scores in Human Renal Cell Carcinoma. Pathol Res Pract., 1991; 187(4): 482-86. doi: 10.1016/s0344-0338(11)80011-7.

[20]Ettinger SN, Scase TJ, Oberthaler KT, Craft DM, McKnight $J A$, et al. Association of argyrophilicnucleolar organizing regions, Ki-67, and proliferating cell nuclear antigen scores with histologic grade and survival in dogs with soft tissue sarcomas: 60 cases (1996-2002), 2006; J Am Vet Med Assoc., 228(7): 1053-62. doi: 10.2460/javma.228.7. 1053.
[21]Jelen M, Blok K, Blok R, Leskow E, Gruszczynska ST. Nucleolus organizer regions (agnors) and Ki-67 antigens expression from the cervix and cervical canal smears with respect to changes in ascus and AGUS (atypical cells of undetermined significance) according to Bethesda Cytological System of Classification. Ginekologia Polska, 2002; 73(12): 1192-98.

[22]Yue L, Iwai M, Furuta I. Evaluation of argyrophilicnucleolar organizer regions in tongue squamous cell carcinomas. Oral Oncol., 1999; 35(1): 70-76. doi: 10.1016/s1368-8375(98)00074-8.

[23] Kamath KP, Vidya M, Shetty, N., Karkera BV, Jogi H. Nucleolar Organizing Regions and $\alpha$-Smooth Muscle Actin Expression in a Case of Ameloblastic Carcinoma. Head and Neck Pathol., 2010; 4(2): 15762. doi: 10.1007/s12105-010-0173-7.

[24]Crocker J, Nar P. Nucleolar organizer regions in lymphomas. J Pathol., 1997; 151(2): 111-18. doi: 10.1002/path.1711510203. 